\title{
Consolidation power of extrinsic rewards: reward cues enhance long-term memory for irrelevant past events
}

Article

Accepted Version

Murayama, K. and Kitagami, S. (2014) Consolidation power of extrinsic rewards: reward cues enhance long-term memory for irrelevant past events. Journal of Experimental Psychology: General, 143 (1). pp. 15-20. ISSN 1939-2222 doi: https://doi.org/10.1037/a0031992 Available at https://centaur.reading.ac.uk/34841/

It is advisable to refer to the publisher's version if you intend to cite from the work. See Guidance on citing.

To link to this article DOI: http://dx.doi.org/10.1037/a0031992

Publisher: American Psychological Association

Publisher statement: This article may not exactly replicate the final version published in the APA journal. It is not the copy of record.

All outputs in CentAUR are protected by Intellectual Property Rights law, including copyright law. Copyright and IPR is retained by the creators or other copyright holders. Terms and conditions for use of this material are defined in the End User Agreement.

www.reading.ac.uk/centaur 


\section{CentAUR}

Central Archive at the University of Reading

Reading's research outputs online 
Consolidation Power of Extrinsic Rewards: Reward Cues Enhance Long-Term Memory for Irrelevant Past Events

\author{
Kou Murayama
}

University of California, Los Angeles

\author{
Shinji Kitagami \\ Nagoya University
}

Journal of Experimental Psychology: General (in press)

Author Note

Kou Murayama, Department of Psychology, University of California, Los Angeles and Japan Society for the Promotion of Science, Japan; Shinji Kitagami, Graduate School of Environmental Studies, Nagoya University, Japan.

The present research was supported by Grant-in-Aid for Scientific Research B\#2133016 (to S. Kitagami). We thank Drs. Dharshan Kumaran (University College London), Alan Castel (University of California, Los Angeles), Bridgid Finn (Washington University in St. Louis), Mara Mather (University of California, Los Angeles), Atsunobu Suzuki (Nagoya University) and Jun Kawaguchi (Nagoya University) for providing helpful comments on an earlier version of this article.

Correspondence concerning this article should be addressed to Kou Murayama, Department of Psychology, University of California, Los Angeles, 1285 Franz Hall Box 951563, Los Angeles, CA 90095, e-mail: murakou@orion.ocn.ne.jp 


\begin{abstract}
Recent research suggests that extrinsic rewards promote memory consolidation through dopaminergic modulation processes. However, no conclusive behavioral evidence exists given that the influence of extrinsic reward on attention and motivation during encoding and consolidation processes are inherently confounded. The present study provides behavioral evidence that extrinsic rewards (i.e., monetary incentives) enhance human memory consolidation independently of attention and motivation. Participants saw neutral pictures, followed by a reward or control cue in an unrelated context. Our results (and a direct replication study) demonstrated that the reward cue predicted a retrograde enhancement of memory for the preceding neutral pictures. This retrograde effect was observed only after a delay, not immediately upon testing. An additional experiment showed that emotional arousal or unconscious resource mobilization cannot explain the retrograde enhancement effect. These results provide support for the notion that the dopaminergic memory consolidation effect can result from extrinsic reward.

Keywords: money, incentives, postencoding, reinforcement learning, retrograde memory enhancement
\end{abstract}


We often encounter more information than we can actually remember; in such situations, our memory system distinguishes things that matter from things that do not by relying on a variety of signals (Castel, 2008; Mather \& Schoeke, 2011). Extrinsic reward is one such signal that has garnered considerable attention. Recent neuroscientific research has suggested that anticipation of an extrinsic reward, such as money, might promote memory consolidation by activating the mesolimbic reward system, which increases phasic dopamine release in the hippocampal memory system (Duzel, Bunzeck, Guitart-Masip, \& Duzel, 2010; Lisman \& Grace, 2005; Shohamy \& Adcock, 2010). This dopaminergic memory consolidation hypothesis is supported by several behavioral experiments: Anticipation of an extrinsic reward enhances memory performance (e.g., Bialleck, et al., 2011; Shigemune, et al., 2010; Weiner, 1966; Wittmann et al., 2005).

However, the evidence is not as compelling as it first appears. Surprisingly little attention has been given to the effects of confounding factors that are critical when dealing with extrinsic rewards: motivation and attention. The dopaminergic memory consolidation hypothesis posits that the reward system directly modulates memory consolidation within the hippocampal memory system; consequently, extrinsic rewards can enhance memory consolidation even without accompanying motivational or attentional processes (Shohamy \& Adcock, 2010). However, the fact that rewards have strong incentive properties inevitably drives people to be engaged in, and pay attention to, learning tasks. Consequently, most past studies showing the memory enhancement effect of extrinsic reward anticipation might be explained in terms of increased motivation and attention: participants might better remember task-related materials simply because they are motivated by the incentive. This confounding problem is 
exacerbated by the fact that most previous studies on this topic have utilized intentional learning paradigms in which participants are explicitly told that rewards are contingent on their memory performance (Adcock, Thangavel, Whitfield-Gabrieli, Knutson, \& Gabrieli, 2006; Harley, 1965; Shigemune, et al., 2010; Thornton, et al., 2007; Wickens \& Simpson, 1968).

A few recent studies have observed memory enhancement effects in incidental learning paradigms (Mather \& Schoeke, 2011; Wittmann, Daw, Seymour, \& Dolan, 2008; Wittmann, et al., 2005). Such findings might provide direct evidence for the dopaminergic memory consolidation hypothesis. In these studies, however, learning materials are still used as cues or as targets for the rewarding task. Therefore, motivation and attention cannot be excluded as contributors to increased memory performance during reward trials. Neuroimaging studies have demonstrated that greater activity in the reward system and the hippocampus during encoding predicts better memory performance (Adcock, et al., 2006; Wittmann, et al., 2005). Such findings might constitute strong evidence for the dopaminergic memory consolidation hypothesis. However, these findings are correlational, and motivation or attention due to a reward may mediate or explain this relationship.

We aim to provide strong behavioral evidence for the dopaminergic memory consolidation hypothesis by showing that extrinsic rewards promote memory consolidation even without motivational or attentional processes. For this purpose, we examined the effects of a reward cue on incidental memory performance for a separate task presented prior to the cue (see Anderson, Wais, \& Gabrieli, 2006). Since learning materials were encoded before the presentation of the reward cue in a completely different context, the reward cue cannot influence encoding. Thus, this design enables 
dissociation of the effects of extrinsic rewards on attention and motivation during encoding from the influence of extrinsic rewards on memory consolidation. We predicted that materials presented prior to the reward cue would be remembered better than those presented prior to a control cue (i.e., retrograde memory enhancement).

We further tested the dopaminergic memory consolidation hypothesis in two ways. First, we compared memory performance immediately after the learning session and after a delay. Hippocampus-dependent memory consolidation is assumed to manifest only after some time has elapsed; this is supported by empirical studies (Murayama \& Kuhbandner, 2011; Sharot \& Phelps, 2004; Sharot \& Yonelinas, 2008). Accordingly, we expect that retrograde memory enhancement would be observed only after a delay, not immediately in the test. Second, we examined the source of individual differences in retrograde memory enhancement. The dopaminergic reward system is generally responsive to rewarding events (Haber \& Knutson, 2010); consequently, individuals with more rewarding outcomes (i.e., high task performance) are expected to have greater overall dopaminergic activation during the task (Gray, 1987; Hahn, et al., 2009). Accordingly, we predict that individual differences in retrograde memory enhancement would be positively related to task performance.

\section{Experiment 1}

\section{Method}

Participants and design. Participants were 33 Japanese undergraduate students (mean age $=18.9$ years). We collected as many observations as we could before the end of the semester. We employed a 2 (Time Interval: immediate test vs. delayed test) $\times 2$ (Postencoding Cue: reward vs. control) factorial design, with the latter being a within-participant factor. 
Materials and procedure. The test stimuli were neutral photographs of real, natural objects (e.g., an apple) and man-made objects (e.g., a ball) taken from Knight and Mather (2009); Lang, Bradley, and Cuthbert (2005); and other resources. There were three lists of 30 photographs. Each list was assigned to a reward cue condition, control cue condition, or foils for the recognition memory test. List assignment was counterbalanced across participants. All tasks were conducted on a computer.

The (incidental) learning session consisted of 60 main trials. Half involved a rewarded task (i.e., reward cue condition), and the other, a control task (i.e., control cue condition). The main trials were presented in random order to prevent carryover effects. Four filler trials were presented at the beginning and end of the experiment.

An experimental trial comprised three consecutive events preceded by a cue indicating the start of the trial: study events, modulator events, and filler events (Figure 1). Study events were neutral events used to probe the effect of a postencoding reward cue presented during the subsequent modulator event. Participants were presented a test stimulus for $2000 \mathrm{~ms}$ and asked to indicate, by pressing a key, whether the picture was natural or man-made (correct response rate $=99.3 \%$ ). Participants were not told that they would be performing a subsequent recognition test.

Modulator events were designed to manipulate postencoding phasic dopamine release for each trial. Dopamine shows phasic activation to brief reward cues (Schultz, 2002; Shohamy, 2011). Accordingly, modulator events started with a 1500-ms cue that signals either a subsequent reward or control task. In the reward task, participants were presented with a stopwatch that started automatically, and the goal was to press a button within 50 ms of a 3-s time point (Murayama, Matsumoto, Izuma, \& Matsumoto, 2010; see also the supplemental materials for a reanalysis of our brain imaging data using this 
task). Participants were told that they would obtain JPY 50 (about USD 0.65) for each successful trial during the stopwatch task; the total amount of money they obtained was visible during the task. For the control task, participants passively viewed a stopwatch and were asked to simply press a button when it automatically stopped (randomly selected between 2800 and $3200 \mathrm{~ms}$ ). No monetary reward was provided during this task.

Filler events were designed to prevent carryover effects (Anderson et al., 2006). Participants performed a response flanker task for $8.0 \mathrm{~s}$ (in addition to a 1.5 -s task cue) wherein they had to determine the direction of the middle of three arrows (correct response rate $=99.1 \%$ ). A filler event comprised a sequence of randomly intermixed four flanker stimuli ( $2 \mathrm{~s}$ each), which were either congruent (the direction of the middle arrow is the same as that of the other arrows) or incongruent (the direction of the middle arrow is not the same as that of the other arrows). Preliminary analysis showed that performance on this task (differences in reaction time between trials with congruent and incongruent stimuli) did not significantly affect recall of the pictures presented in the same or the next trials.

After the learning session, participants in the immediate test condition completed a surprise memory test. Ninety stimuli from all the lists were presented randomly. Participants indicated whether the test stimuli had been presented in the learning session (old) or not (new). Participants in the delayed memory test condition were scheduled to return for an unrelated experiment about 1 week subsequently. The procedure for the delayed memory test was the same as that used in the immediate memory test. A post-experimental question indicated that no participants expected the memory test.

\section{Results and Discussion}


As expected, recognition memory performance (hit rate) was enhanced in the reward cue condition only after a delay (Figure 2). A 2 (Time Interval: immediate test vs. delayed test $) \times 2$ (Postencoding Cue: reward vs. control) mixed model ANOVA revealed a significant main effect of Time Interval, $F(1,31)=57.15, \eta^{2}{ }_{G}=.63, p<.01$, indicating that participants showed better performance on the immediate test than on the delayed test. Importantly, there was a significant Time Interval $\times$ Postencoding Cue interaction, $F(1,31)=6.02, \eta_{G}^{2}=.01, p<.05$. Simple main effect analyses revealed that recognition memory performance in the reward cue condition was better than the control cue condition on the delayed test, $F(1,31)=6.27, p<.05, d=0.54$, but not on the immediate test, $p=.34$. This indicates that postencoding reward cues enhanced memory performance only after a delay ${ }^{1}$. The false alarm rate was significantly higher in the delayed memory test $(M=0.08 ; S D=0.05)$ than in the immediate memory test $(M=0.04, S D=0.02), t(31)=2.84, p<.01, d=1.14$.

To investigate whether individual differences in memory performance were related to task performance, we first determined the memory benefit of postencoding reward cues for each participant by subtracting the recognition performance in the control cue condition from that in the reward cue condition. The resulting score was then correlated with performance on the stopwatch task (i.e., rate of successful trials; $M$ $=.57)$. A positive correlation was found in the delayed test condition $(r=.76, p<.01$, Figure 3), supporting our prediction that high-performing participants (i.e., participants who are supposed to have high overall dopaminergic activation) would demonstrate a stronger retrograde memory consolidation effect in response to the reward cue. In contrast, the correlation in the immediate test condition was not significant $(r=-.12, p$ $=.65)$. 


\section{Experiment 2}

Experiment 1 suggested that postencoding reward cues directly enhance the memory consolidation process, perhaps via dopaminergic activation. However, our reward task (i.e., stopwatch task) may also have enhanced emotional arousal, which in turn could have produced the retrograde memory enhancement effect. The effect of postencoding emotional arousal has been observed elsewhere (Anderson, et al., 2006; Finn \& Roediger, 2011; Knight \& Mather, 2009). Therefore, it would be important to show that emotional arousal is not a viable explanation for our findings (see also the supplemental materials for a reanalysis of our brain imaging data to further examine this possibility). Another alternative explanation is that unconscious resource mobilization may have contributed to the memory enhancement effect. Previous studies have shown that rewards unconsciously recruit executive functioning when a task is demanding (Bijleveld, Custers, \& Aarts, 2009, 2012). Given that our reward task is more demanding than the control task, such resource mobilization might have strengthened the postencoding process, despite little evidence that postencoding effortful processing can enhance memory consolidation.

Experiment 2 was designed to test these alternative explanations. Specifically, we negatively framed the modulator events such that participants would lose money when they failed at the stopwatch task. With this modification, the stopwatch task cue is a punishment cue that signals potential loss of money and serves as a negative emotional event. Importantly, as per the emotional arousal account, retrograde memory enhancement is expected because negative events should also evoke emotional arousal (even more strongly than positive events; see Ito, Larsen, Smith, \& Cacioppo, 1998). In fact, most studies on postencoding emotional arousal found memory enhancement 
effects by using negative arousing stimuli (Finn \& Roediger, 2011; Knight \& Mather, 2009). In addition, because the demand of the reward (punishment) task is the same as that in Experiment 1 (i.e., the same task is used), as per the resource mobilization account, a retrograde memory consolidation effect is also expected. Conversely, researchers have shown that punishment/avoidance learning is related to serotonin, which suppresses the dopaminergic system (for a review, see Dayan \& Huys, 2009). In fact, extant fMRI studies indicate that activation in the dopaminergic reward system is much weaker in the context of punishment/avoidance learning (e.g., Kim, Shimojo, \& O’Doherty, 2006; Robinson, Frank, Sahakian, \& Cools, 2010; Schlund, Magee, \& Hudgins, 2011). Therefore, we expect that the postencoding memory consolidation effect would not be observed when the task cue signals potential loss of money.

Participants and design. In this experiment, we employed only a delayed memory test as the memory consolidation effect was observed only after a delay. Therefore, the experiment employed a one-factor (Postencoding Cue: punishment vs. control) within-participant design. The sample comprised 46 Japanese undergraduate students (mean age $=18.5$ years). As our primary hypothesis may involve the absence of the effect, to minimize the Type II error rate, we determined our sample size by a priori power analysis (Cohen, 1988) based on the effect size obtained in Experiment 1, with $\alpha$ at .05 (two-tailed) and power at .95 .

Materials and procedure. The experimental procedure was the same as that in Experiment 1, with two exceptions. First, we employed only a delayed memory test as described above. Second, before the experiment, participants were provided with a lump sum of JPY 1600 ( USD 20.80; the maximum possible gain in Experiment 1). Participants were instructed that they would lose JPY 50 for each failure on the 
stopwatch task, and the total amount of money lost was displayed during the task. Correct response rates for the picture judgment task and flanker task were comparable with those in Experiment 1 (99.4\% and 99.0\%, respectively). Similar to Experiment 1, reaction time performance on the flanker task did not significantly affect recall of the pictures presented in the same or the next trials.

\section{Results and Discussion}

Recognition memory performance did not significantly differ between the punishment cue condition $(M=.55, S D=.16)$ and control cue condition $(M=.55, S D$ $=.17), t(45)=0.04, p=0.97, d=-0.01^{2}$. These results are inconsistent with the possibility that emotional arousal or resource mobilization played a role in the retrograde memory enhancement effect observed in Experiment 1. The correlation between task performance and differences in memory performance between the conditions was not significant, $r=.01, p=.96$.

\section{General Discussion}

Despite the recent attention paid to the dopaminergic memory consolidation hypothesis, most previous studies failed to control for attention and motivation, substantially limiting the interpretation of their findings. The current study addressed this critical issue by demonstrating that the presentation of an irrelevant reward cue following a test stimulus can enhance recognition memory of that stimulus. The effect was observed only after a delay, and the memory enhancement effect was positively correlated with the total amount of obtained monetary reward. Taken together, these results provide strong support for the dopaminergic memory consolidation effect of extrinsic rewards. We also showed that postencoding memory consolidation does not occur when the task is framed as a monetary loss, suggesting that emotional arousal or 
unconscious resource mobilization is not a viable explanation for our findings. Our punishment cues might have created a negative mood that counteracted the positive consolidation effect. Despite little evidence that trial-by-trial negative mood fluctuation has retrograde downstream effects, this possibility should be addressed in future studies.

Our findings have implications for various fields. First, research on motivation has long assumed that extrinsic reward, especially monetary reward, is a powerful motivator. Many previous studies have shown that monetary incentives have a strong motivational influence on various behavioral and psychological processes (Lea \& Webley, 2006; Pessiglione et al., 2007). However, we suggest that extrinsic reward also has a non-motivational influence (see also Vohs, Mead, \& Goode, 2008). Second, the involvement of dopamine in learning has mainly been discussed in the context of model-free reinforcement learning (Dayan \& Niv, 2008; Montague \& Berns, 2002). In this framework, extrinsic rewards activate dopamine release in order to effectively form stimulus-outcome associations. Given our findings that extrinsic rewards consolidate episodic memory, however, it would be important to incorporate the role of the episodic memory system in reinforcement learning (see Lengyel \& Dayan, 2007). In addition, it would be interesting to investigate what components of episodic memory (e.g., recollection vs. familiarity; Yonelinas, 2001) contribute to retrograde memory consolidation.

Finally, our findings highlight the importance of the postencoding period in memory models (Hamann, 2001). Most of the extant memory models focus on the encoding and retrieval periods as critical parameters to determine memory performance. In contrast, our results suggest that even an extraneous stimulus presented after memory encoding could enhance memory performance. Future research should incorporate such 
factors, to establish a more comprehensive model of human memory. 


\section{References}

Adcock, R. A., Thangavel, A., Whitfield-Gabrieli, S., Knutson, B., \& Gabrieli, J. D. E. (2006). Reward-motivated learning: Mesolimbic activation precedes memory formation. Neuron, 50, 507-517. doi: 10.1016/j.neuron.2006.03.036

Anderson, A. K., Wais, P. E., \& Gabrieli, J. D. E. (2006). Emotion enhances remembrance of neutral event past. Proceedings of the National Academy of Sciences of the United States of America, 103, 1599-1604. doi: 10.1038/nn1413

Bialleck, K. A., Schaal, H.-P., Kranz, T. A., Fell, J., Elger, C. E., \& Axmacher, N. (2011). Ventromedial prefrontal cortex activation is associated with memory formation for predictable rewards. PLoS ONE, 6, e16695. doi: 10.1371/journal.pone.0016695

Bijleveld, E., Custers, R., \& Aarts, H. (2009). The unconscious eyeopener: Pupil size reveals strategic recruitment of resources upon presentation of subliminal reward cues. Psychological Science, 20, 1313-1315. doi:10.1111/j.1467-9280.2009.02443.x

Bijleveld, E., Custers, R., \& Aarts, H. (2012). Human reward pursuit: From rudimentary to higher-level functions. Current Directions in Psychological Science, 21, 194-199. doi: 10.1177/0963721412438463

Castel, A. D. (2008). The adaptive and strategic use of memory by older adults: Evaluative processing and value-directed remembering. In A. S. Benjamin \& B. H. Ross (Eds.), The psychology of learning and motivation, vol. 48 (pp. 225-270). London: Academic Press.

Cohen, J. (1988). Statistical power analysis for the behavioral sciences (2nd ed.). Hillsdale, NJ: Erlbaum. 
Dayan, P., and Huys, Q. J. M. (2009). Serotonin in affective control. Annual Review of Neuroscience, 32, 95-126. doi: 10.1146/annurev.neuro.051508.135607

Dayan, P., \& Niv, Y. (2008). Reinforcement learning and the brain: The good, the bad, and the ugly. Current Opinion in Neurobiology, 18, 185-196. doi: 10.1016/j.conb.2008.08.003

Duzel, E., Bunzeck, N., Guitart-Masip, M., \& Duzel, S. (2010). Novelty-related motivation of anticipation and exploration by dopamine (nomad): Implications for healthy aging. Neuroscience and Biobehavioral Reviews, 34, 660-669. doi: 10.1016/j.neubiorev.2009.08.006

Finn, B., \& Roediger, H. L., III. (2011). Enhancing retention through reconsolidation: Negative emotional arousal following retrieval enhances later recall. Psychological Science, 22, 781-786. doi: 10.1177/0956797611407932

Gray, J. A. (1987). The psychology of fear and stress (2nd edition). Cambridge: Cambridge University Press.

Hahn, T., Dresler, T., Ehlis, A. C., Plichta, M. M., Heinzel, S., Polak, T., . . Fallgatter, A. J. (2009). Neural response to reward anticipation is modulated by Gray's impulsivity. NeuroImage, 46, 1148-1153. doi: 10.1016/j.neuroimage.2009.03.038

Hamann, S. (2001). Cognitive and neural mechanism of emotional memory. Trends in Cognitive Sciences, 5, 394-400. doi: 10.1016/S1364-6613(00)01707-1

Haber, S. N., \& Knutson, B. (2010). The reward circuit: Linking primate anatomy and human imaging. Neuropsychopharmacology, 35, 4-26. doi: 10.1038/npp.2009.129

Harley, J. W. F. (1965). The effect of monetary incentive in paired associate learning 
using a differential method. Psychonomic Science, 2, 377-378.

Ito, T. A., Larsen, J. T., Smith, N. K., \& Cacioppo, J. T. (1998). Negative information weighs more heavily on the brain: The negativity bias in evaluative categorizations. Journal of Personality and Social Psychology, 75, 887-900. doi: $10.1037 / 0022-3514.75 .4 .887$

Kim, H., Shimojo, S., \& O'Doherty, J. P. (2006). Is avoiding an aversive outcome rewarding? Neural substrates of avoidance learning in the human brain. PLoS Biology, 4. doi: :10.1371/journal.pbio.0040233

Knight, M., \& Mather, M. (2009). Reconciling findings of emotion-induced memory enhancement and impairment of preceding items. Emotion, 9, 763-781. doi: $10.1037 / \mathrm{a} 0017281$

Lang, P. J., Bradley, M. M., \& Cuthbert, B. N. (2005). International Affective Picture System (IAPS): Digitized photographs, instruction manual, and affective ratings (Tech. Rep. No. A-6). Gainesville: University of Florida, Center for Research in Psychophysiology.

Lea, S. E. G., \& Webley, P. (2006). Money as tool, money as drug: The biological psychology of a strong incentive. Behavioral and Brain Sciences, 29, 161-209. doi: doi:10.1017/S0140525X06009046

Lengyel, M., \& Dayan, P. (2008). Hippocampal contributions to control: the third way. Advances in Neural Information Processing Systems, 20, 889-896.

Lisman, J. E., \& Grace, A. A. (2005). The hippocampal-VTA loop: Controlling the entry of information into long-term memory. Neuron, 46, 703-713. doi: 10.1016/j.neuron.2005.05.002

Mather, M., \& Schoeke, A. (2011). Positive outcomes enhance incidental learning for 
both younger and older adults. Frontiers in Neuroscience, 5. doi: 10.3389/fnins.2011.00129

Montague, P. R., \& Berns, G. S. (2002). Neural economics and the biological substrates of valuation. Neuron, 36, 265-284. doi: 10.1016/S0896-6273(02)00974-1

Murayama, K., \& Kuhbandner, C. (2011). Money enhances memory consolidation—but only for boring material. Cognition, 119, 120-124. doi: 10.1016/j.cognition.2011.01.001

Murayama, K., Matsumoto, M., Izuma, K., \& Matsumoto, K. (2010). Neural basis of the undermining effect of monetary reward on intrinsic motivation. Proceedings of the National Academy of Sciences of the United States of America, 107, 20911-20916. doi: 10.1073/pnas.1013305107

Pessiglione, M., Schmidt, L., Draganski, B., Kalisch, R., Lau, H., Dolan, R. J., \& Frith, C. D. (2007). How the brain translates money into force: A neuroimaging study of subliminal motivation. Science, 316, 904-906. doi: 10.1126/science.1140459

Robinson, O. J., Frank, M. J., Sahakian, B. J., \& Cools, R. (2010). Dissociable responses to punishment in distinct striatal regions during reversal learning. Neuroimage, 51, 1459-1467. doi: 10.1016/j.neuroimage.2010.03.036

Schlund, M. W., Magee, S., \& Hudgins, C. D. (2011). Human avoidance and approach learning: overlapping neural systems and experiential avoidance modulation of frontal and limbic responses. Behavioural Brain Research. 225, 437-48. doi: 10.1016/j.bbr.2011.07.054

Schultz, W. (2002). Getting formal with dopamine and reward. Neuron, 36, 241-263. doi: 10.1016/S0896-6273(02)00967-4

Sharot, T., \& Phelps, E. A. (2004). How arousal modulates memory: Disentangling the 
effects of attention and retention. Cognitive, Affective \& Behavioral Neuroscience, 4, 294-306. doi: 10.3758/cabn.4.3.294

Sharot, T. \& Yonelinas A. P. (2008). Differential time-dependent effects of emotion on the recollective experience and memory for contextual information. Cognition, 106, 538-547. doi: 10.1016/j.cognition.2007.03.002

Shigemune, Y., Abe, N., Suzuki, M., Ueno, A., Mori, E., Tashiro, M... Fujii, T. (2010). Effects of emotion and reward motivation on neural correlates of episodic memory encoding: A PET study. Neuroscience Research, 67, 72-79. doi: 10.1016/j.neures.2010.01.003

Shohamy, D. (2011). Learning and motivation in the human striatum. Current Opinion in Neurobiology, 21, 408-414. doi: 10.1016/j.conb.2011.05.009

Shohamy, D., \& Adcock, R. A. (2010). Dopamine and adaptive memory. Trends in Cognitive Sciences, 14, 464-472. doi: 10.1016/j.tics.2010.08.002

Thornton, A. E., Boudreau, V. G., Griffiths, S. Y., Woodward, T. S., Fawkes-Kirby, T., \& Honer, W. G. (2007). The impact of monetary reward on memory in schizophrenia spectrum disorder. Neuropsychology, 21, 631-645. doi: 10.1037/0894-4105.21.5.631

Vohs, K. D., Mead, N. L., \& Goode, M. R. (2008). Merely activating the concept of money changes personal and interpersonal behavior. Current Directions in Psychological Science, 17, 208-212. doi: 10.1111/j.1467-8721.2008.00576.x

Weiner, B. (1966). Effects of motivation on the availability and retrieval of memory traces. Psychological Bulletin, 65, 24-37. doi: 10.1037/h0022664

Wickens, D. D., \& Simpson, C. K. (1968). Trace cue position, motivation, and short-term memory. Journal of Experimental Psychology, 76, 282-285. doi: 


\section{$10.1037 / \mathrm{h} 0025386$}

Wittmann, B. C., Daw, N. D., Seymour, B., \& Dolan, R. J. (2008). Striatal activity underlies novelty-based choice in humans. Neuron, 58, 967-973. doi: 10.1016/j.neuron.2008.04.027

Wittmann, B. C., Schott, B. H., Guderian, S., Frey, J. U., Heinze, H. J., \& Duzel, E. (2005). Reward-related fMRI activation of dopaminergic midbrain is associated with enhanced hippocampus-dependent long-term memory formation. Neuron, 45, 459-467. doi: 10.1016/j.neuron.2005.01.010

Yonelinas, A. P. (2001). Components of episodic memory: the contribution of recollection and familiarity. Philosophical Transactions of the Royal Society of London. Series B: Biological Sciences, 356, 1363-1374. doi:

10.1098/rstb.2001.0939 


\section{Footnotes}

${ }^{1}$ As recommended by a reviewer, we conducted a direct replication study to confirm that our main findings were not false positives. We ran exactly the same experiment with only the delayed memory condition, as this is the most critical condition in our study. Again, we collected as many observations as we could before the end of the semester, resulting in a sample of 34 participants after excluding one participant who expected the memory test (see Murayama \& Kuhbandner, 2011). The recognition hit rate was better during the reward cue condition than the control cue condition, $t(33)=$ $2.08, p<.05, d=0.36$. We combined the data from the replication study with those from the original study, and the results were still significant, $t(49)=2.81, p<.01$. Because this is the only replication study that we ran, the combined Type-I error rate across the two studies is less than 0.0025 .

${ }^{2}$ These mean hit rates do not significantly differ from those in the control cue condition from the pooled data $(N=50)$ of Experiment 1 and the replication study ( $p \mathrm{~s}>.14)$. 


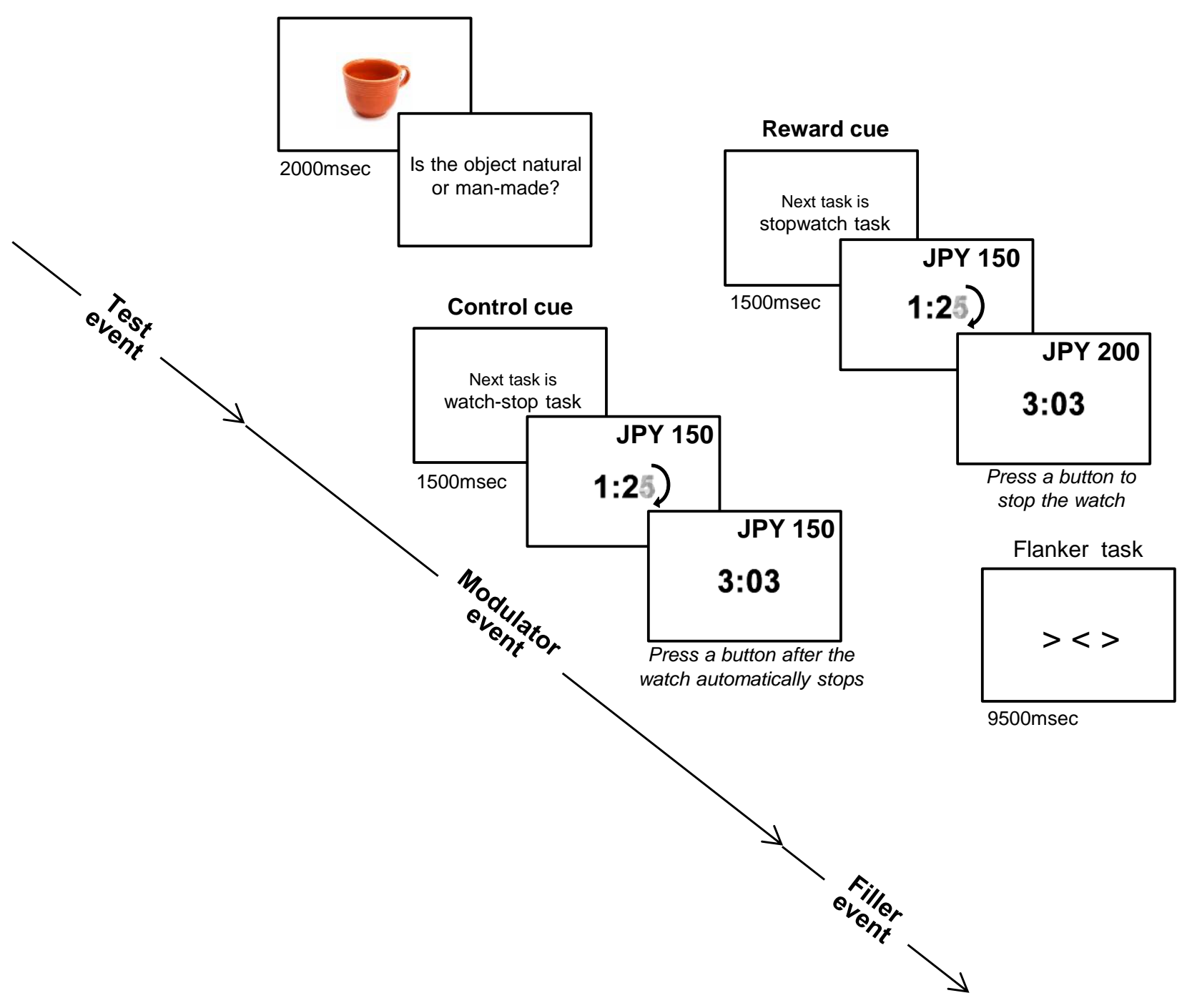

Figure 1. A schematic representation of the trial sequence. 


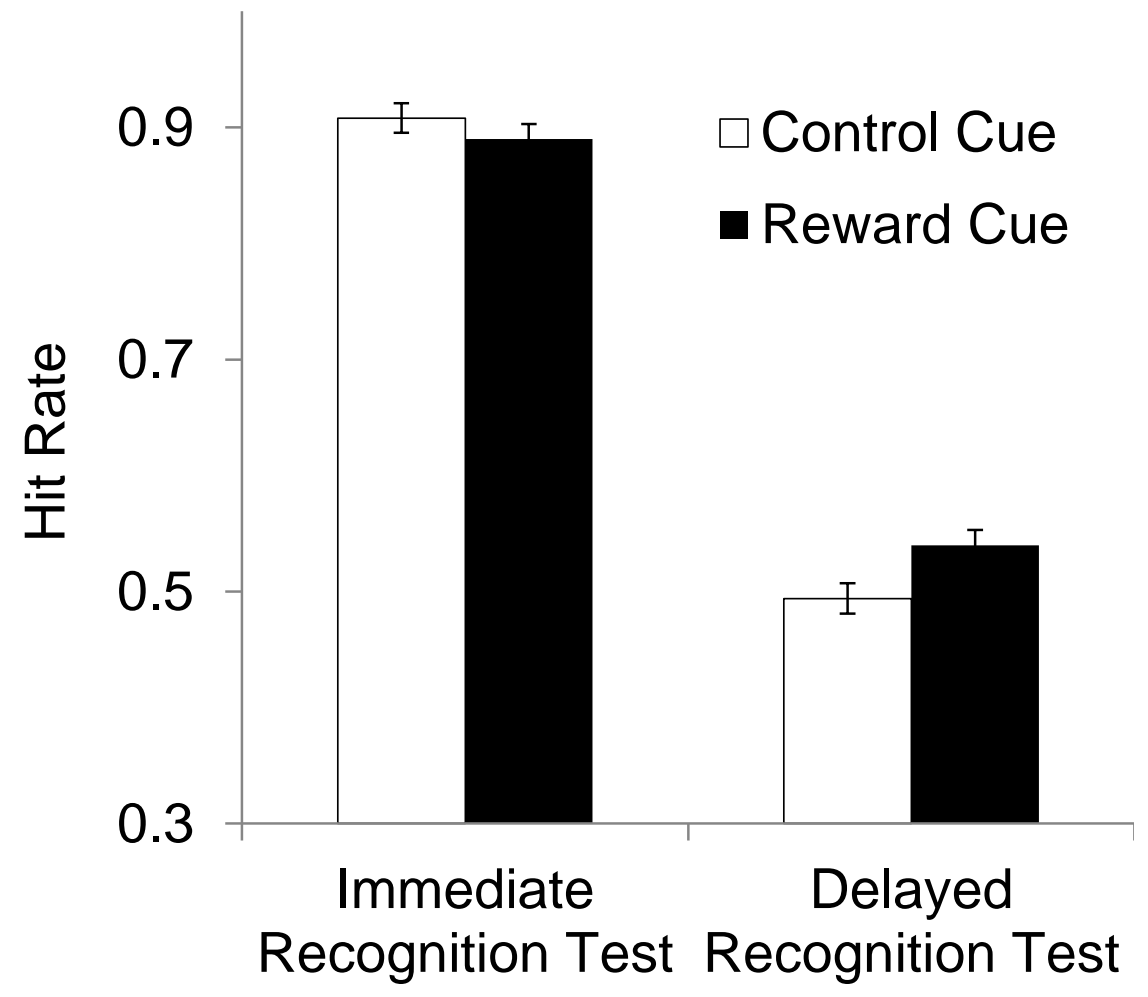

Figure 2. Recognition memory performance (hit rate) as a function of postencoding reward cue and time interval. $* p<.05$. 


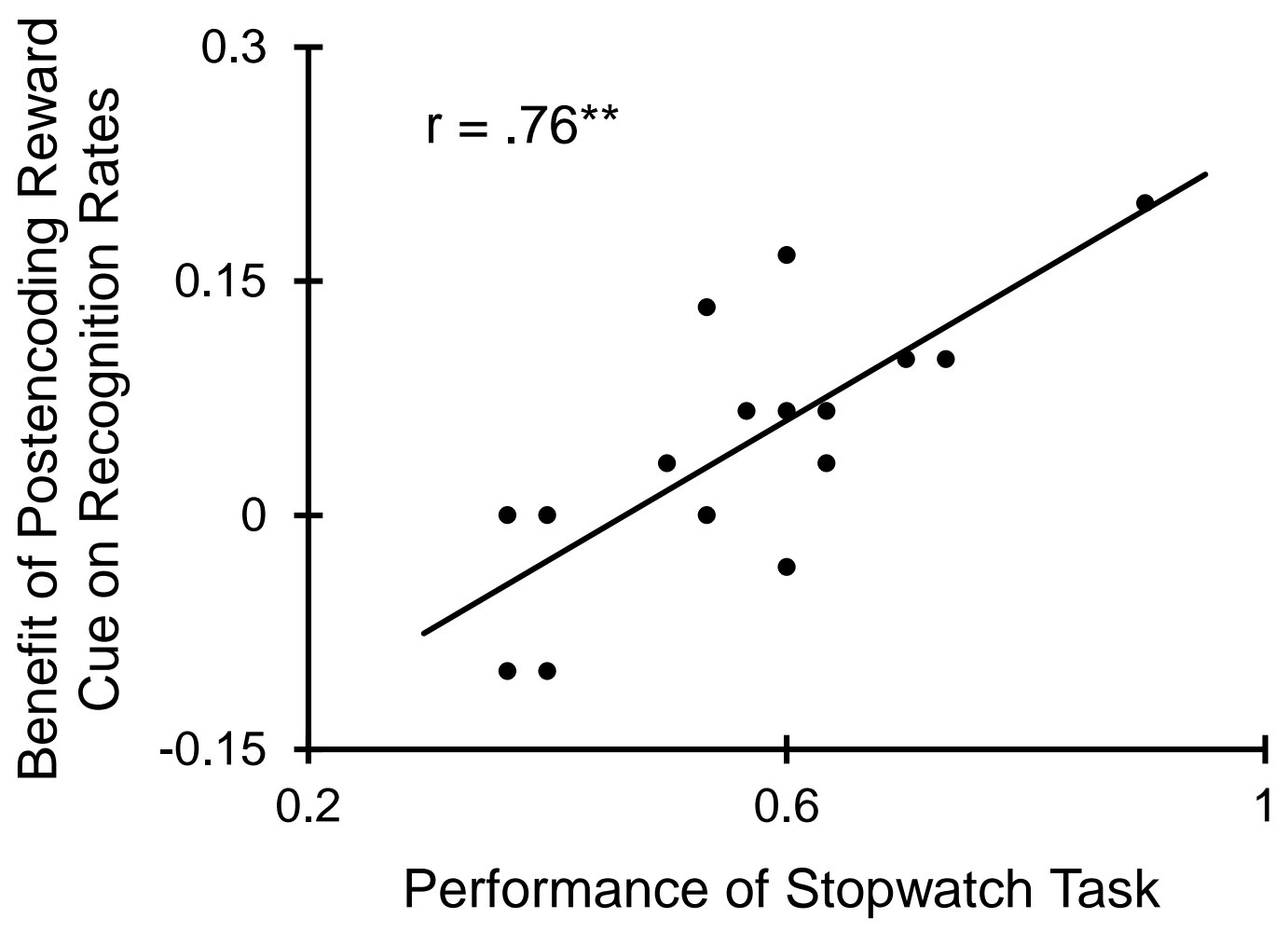

Figure 3. Scatter plot of the overall performance of the stopwatch task and the benefit of the postencoding reward cue on recognition rates (as defined by the memory performance difference between the reward cue and the control cue conditions). $* * p$ $<.01$. 